TRANSACTIONS OF THE

AMERICAN MATHEMATICAL SOCIETY

Volume 362, Number 7, July 2010, Pages 3757-3777

S 0002-9947(10)05008-7

Article electronically published on February 12, 2010

\title{
SOME REGULAR SYMMETRIC PAIRS
}

\author{
AVRAHAM AIZENBUD AND DMITRY GOUREVITCH
}

\begin{abstract}
In an earlier paper we explored the question what symmetric pairs are Gelfand pairs. We introduced the notion of regular symmetric pair and conjectured that all symmetric pairs are regular. This conjecture would imply that many symmetric pairs are Gelfand pairs, including all connected symmetric pairs over $\mathbb{C}$.

In this paper we show that the pairs $(G L(V), O(V)),(G L(V), U(V))$, $(U(V), O(V)),(O(V \oplus W), O(V) \times O(W)),(U(V \oplus W), U(V) \times U(W))$ are regular, where $V$ and $W$ are quadratic or Hermitian spaces over an arbitrary local field of characteristic zero. We deduce from this that the pairs $\left(G L_{n}(\mathbb{C}), O_{n}(\mathbb{C})\right)$ and $\left(O_{n+m}(\mathbb{C}), O_{n}(\mathbb{C}) \times O_{m}(\mathbb{C})\right)$ are Gelfand pairs.
\end{abstract}

\section{Contents}

1. Introduction

1.1. Structure of the paper

2.1. Gelfand pairs

2.2. Symmetric pairs

3. Main results

4. $\mathbb{Z} / 2 \mathbb{Z}$ graded representations of $s l_{2}$ and their defects 3765

4.1. Graded representations of $s l_{2} \quad 3765$

4.2. Defects

5. Proof of regularity and tameness

5.1. The pair $(G L(V), O(V))$

5.3. The pairs $(G L(V), U(V)),\left(U\left(V_{1} \oplus V_{2}\right), U\left(V_{1}\right) \times U\left(V_{2}\right)\right)$ and $\left(U\left(V_{D}\right), O(V)\right)$

6. Computation of descendants

6.1. Preliminaries and notation for orthogonal and unitary groups $\quad 3769$

6.2. The pair $(G L(V), O(V)) \quad 3770$

6.3. The pair $(G L(V), U(V))$

Received by the editors September 11, 2008.

2010 Mathematics Subject Classification. Primary 20G05, 20G25, 22 E45.

Key words and phrases. Uniqueness, multiplicity one, Gelfand pair, symmetric pair, unitary group, orthogonal group.

Both authors were partially supported by a BSF grant, a GIF grant, and an ISF Center of excellency grant. The first author was also supported by ISF grant No. 583/09 and the second author by NSF grant DMS-0635607. Any opinions, findings and conclusions or recommendations expressed in this material are those of the authors and do not necessarily reflect the views of the National Science Foundation.

(C)2010 American Mathematical Society Reverts to public domain 28 years from publication 
6.4. The pair $\left(U\left(V_{D}\right), O(V)\right) \quad 3771$

6.5. The pair $\left(O\left(V_{1} \oplus V_{2}\right), O\left(V_{1}\right) \times O\left(V_{2}\right)\right)$

6.6. The pair $\left(U\left(V_{1} \oplus V_{2}\right), U\left(V_{1}\right) \times U\left(V_{2}\right)\right)$

6.7. Genealogical trees of the symmetric pairs considered 3776

Acknowledgements 3777

References 3777

\section{INTRODUCTION}

In AG2 we explored the question what symmetric pairs are Gelfand pairs. We introduced the notion of regular symmetric pair and conjectured that all symmetric pairs are regular. This conjecture would imply that many symmetric pairs are Gelfand pairs, including all connected symmetric pairs over $\mathbb{C}$.

In this paper we show that the pairs $(G L(V), O(V)),(G L(V), U(V))$, $(U(V), O(V)),(O(V \oplus W), O(V) \times O(W)),(U(V \oplus W), U(V) \times U(W))$ are regular, where $V$ and $W$ are quadratic or Hermitian spaces over an arbitrary local field of characteristic zero. We deduce from this that the pairs $\left(G L_{n}(\mathbb{C}), O_{n}(\mathbb{C})\right)$ and $\left(O_{n+m}(\mathbb{C}), O_{n}(\mathbb{C}) \times O_{m}(\mathbb{C})\right)$ are Gelfand pairs.

In general, if we would know that all symmetric pairs are regular, then in order to show that a given symmetric pair $(G, H)$ is a Gelfand pair it would be enough to check the following condition that we called "goodness":

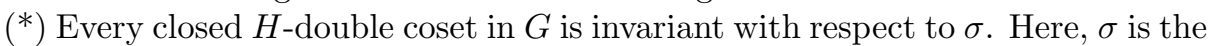
anti-involution defined by $\sigma(g):=\theta\left(g^{-1}\right)$ and $\theta$ is an involution (i.e. automorphism of order 2) of $G$ such that $H=G^{\theta}$.

This condition always holds for connected symmetric pairs over $\mathbb{C}$.

Meanwhile, before the conjecture is proven, in order to show that a given symmetric pair is a Gelfand pair, one has to verify that the pair is good, to prove that it is regular and also to compute its "descendants" and show that they are also regular. The "descendants" are certain symmetric pairs related to centralizers of semisimple elements.

In this paper we develop further the tools from AG2 for proving regularity of symmetric pairs. We also introduce a systematic way to compute descendants of classical symmetric pairs.

Based on that we show that all the descendants of the above symmetric pairs are regular.

1.1. Structure of the paper. In section 2 we introduce the notions that we discuss in this paper. In subsection 2.1] we discuss the notion of a Gelfand pair and review a classical technique for proving the Gelfand property due to Gelfand and Kazhdan. In subsection 2.2 we review the results of [AG2], introduce the notions of symmetric pair, descendants of a symmetric pair, good symmetric pair and regular symmetric pair mentioned above and discuss their relations to the Gelfand property.

In section 3 we formulate the main results of the paper. We also explain how they follow from the rest of the paper.

In section 4 we introduce terminology that enables us to prove regularity for the symmetric pairs in question.

In section 5 we prove regularity for the symmetric pairs in question. 
In section 6 we compute the descendants of those symmetric pairs.

\section{Preliminaries And NOtation}

- Throughout the paper we fix an arbitrary local field $F$ of characteristic zero.

- All the algebraic varieties and algebraic groups that we will consider will be defined over $F$.

- For a group $G$ acting on a set $X$ and an element $x \in X$ we denote by $G_{x}$ the stabilizer of $x$.

- By a reductive group we mean an algebraic reductive group.

In this paper we will refer to distributions on algebraic varieties over Archimedean and non-Archimedean fields. In the non-Archimedean case we mean the notion of distributions on $l$-spaces from [BZ], that is, linear functionals on the space of locally constant compactly supported functions. In the Archimedean case one can consider the usual notion of distributions, that is, continuous functionals on the space of smooth compactly supported functions, or the notion of Schwartz distributions (see e.g. [AG1]). It does not matter here which notion to choose since in the cases of consideration of this paper, if there are no nonzero equivariant Schwartz distributions, then there are no nonzero equivariant distributions at all (see Theorem 4.0.2 in AG2]).

Notation 2.0.1. Let $E$ be an extension of $F$. Let $G$ be an algebraic group defined over $F$. We denote by $G_{E / F}$ the canonical algebraic group defined over $F$ such that $G_{E / F}(F)=G(E)$.

2.1. Gelfand pairs. In this section we recall a technique due to Gelfand and Kazhdan ( $\mathrm{GK}]$ ) which allows us to deduce statements in representation theory from statements on invariant distributions. For a more detailed description, see AGS, section 2 .

Definition 2.1.1. Let $G$ be a reductive group. By an admissible representation of $G$ we mean an admissible representation of $G(F)$ if $F$ is non-Archimedean (see [BZ] and an admissible smooth Fréchet representation of $G(F)$ if $F$ is Archimedean.

We now introduce three notions of Gelfand pair.

Definition 2.1.2. Let $H \subset G$ be a pair of reductive groups.

- We say that $(G, H)$ satisfy GP1 if for any irreducible admissible representation $(\pi, E)$ of $G$ we have

$$
\operatorname{dim} \operatorname{Hom}_{H(F)}(E, \mathbb{C}) \leq 1 .
$$

- We say that $(G, H)$ satisfy GP2 if for any irreducible admissible representation $(\pi, E)$ of $G$ we have

$$
\operatorname{dim} \operatorname{Hom}_{H(F)}(E, \mathbb{C}) \cdot \operatorname{dim} \operatorname{Hom}_{H}(\widetilde{E}, \mathbb{C}) \leq 1 .
$$

- We say that $(G, H)$ satisfy GP3 if for any irreducible unitary representation $(\pi, \mathcal{H})$ of $G(F)$ on a Hilbert space $\mathcal{H}$ we have

$$
\operatorname{dim} \operatorname{Hom}_{H(F)}\left(\mathcal{H}^{\infty}, \mathbb{C}\right) \leq 1 .
$$


Property GP1 was established by Gelfand and Kazhdan in certain $p$-adic cases (see [GK]). Property GP2 was introduced in Gro in the $p$-adic setting. Property GP3 was studied extensively by various authors under the name generalized Gelfand pair both in the real and $p$-adic settings (see e.g. $[\mathrm{vD}, \mathrm{BvD}]$ ).

We have the following straightforward proposition.

Proposition 2.1.3. $G P 1 \Rightarrow G P 2 \Rightarrow G P 3$.

We will use the following theorem from AGS, which is a version of a classical theorem of Gelfand and Kazhdan.

Theorem 2.1.4. Let $H \subset G$ be reductive groups and let $\tau$ be an involutive antiautomorphism of $G$ and assume that $\tau(H)=H$. Suppose $\tau(\xi)=\xi$ for all $H(F)$ bi-invariant distributions $\xi$ on $G(F)$. Then $(G, H)$ satisfies GP2.

In the cases we consider in this paper, GP2 is equivalent to GP1 by the following proposition.

\section{Proposition 2.1.5.}

(i) Let $V$ be a quadratic space (i.e. a linear space with a nondegenerate quadratic form) and let $H \subset G L(V)$ be any transpose invariant subgroup. Then GP1 is equivalent to GP2 for the pair $(\mathrm{GL}(V), H)$.

(ii) Let $V$ be a quadratic space and let $H \subset O(V)$ be any subgroup. Then GP1 is equivalent to GP2 for the pair $(O(V), H)$.

This follows from the following 2 propositions.

Proposition 2.1.6. Let $H \subset G$ be reductive groups and let $\tau$ be an anti-automorphism of $G$ such that

(i) $\tau^{2} \in \operatorname{Ad}(G(F))$;

(ii) $\tau$ preserves any closed conjugacy class in $G(F)$;

(iii) $\tau(H)=H$.

Then GP1 is equivalent to GP2 for the pair $(G, H)$.

For the proof, see AG2, Corollary 8.2.3.

\section{Proposition 2.1.7.}

(i) Let $V$ be a quadratic space and let $g \in G L(V)$. Then $g$ is conjugate to $g^{t}$.

(ii) Let $V$ be a quadratic space and let $g \in O(V)$. Then $g$ is conjugate to $g^{-1}$ inside $O(V)$.

Part (i) is well known. For the proof of (ii), see [MVW], Proposition I.2 in chapter 4 .

2.2. Symmetric pairs. In this subsection we review some tools developed in AG2 that enable us to prove that a symmetric pair is a Gelfand pair. The main results discussed in this subsection are Theorem 2.2.16, Theorem 2.2.24 and Proposition 2.2 .19

Definition 2.2.1. A symmetric pair is a triple $(G, H, \theta)$, where $H \subset G$ are reductive groups, and $\theta$ is an involution of $G$ such that $H=G^{\theta}$. We call a symmetric pair connected if $G / H$ is connected.

For a symmetric pair $(G, H, \theta)$ we define an anti-involution $\sigma: G \rightarrow G$ by $\sigma(g):=\theta\left(g^{-1}\right)$, denote $\mathfrak{g}:=\operatorname{Lie} G, \mathfrak{h}:=$ LieH, $\mathfrak{g}^{\sigma}:=\{a \in \mathfrak{g} \mid \theta(a)=-a\}$. Note 
that $H$ acts on $\mathfrak{g}^{\sigma}$ by the adjoint action. Denote also $G^{\sigma}:=\{g \in G \mid \sigma(g)=g\}$ and define a symmetrization map $s: G \rightarrow G^{\sigma}$ by $s(g):=g \sigma(g)$.

In the case when the involution is obvious we will omit it.

Remark 2.2.2. Let $(G, H, \theta)$ be a symmetric pair. Then $\mathfrak{g}$ has a $\mathbb{Z} / 2 \mathbb{Z}$ grading given by $\theta$.

Definition 2.2.3. Let $\left(G_{1}, H_{1}, \theta_{1}\right)$ and $\left(G_{2}, H_{2}, \theta_{2}\right)$ be symmetric pairs. We define their product to be the symmetric pair $\left(G_{1} \times G_{2}, H_{1} \times H_{2}, \theta_{1} \times \theta_{2}\right)$.

Definition 2.2.4. We call a symmetric pair $(G, H, \theta) \operatorname{good}$ if for any closed $H(F) \times$ $H(F)$ orbit $O \subset G(F)$, we have $\sigma(O)=O$.

Proposition 2.2.5. Every connected symmetric pair over $\mathbb{C}$ is good.

For the proof, see e.g. AG2, Corollary 7.1.7.

Definition 2.2.6. We say that a symmetric pair $(G, H, \theta)$ is a GK pair if any $H(F) \times H(F)$-invariant distribution on $G(F)$ is $\sigma$-invariant.

Remark 2.2.7. Theorem 2.1.4 implies that any GK pair satisfies GP2.

2.2.1. Descendants of symmetric pairs.

Proposition 2.2.8. Let $(G, H, \theta)$ be a symmetric pair. Let $g \in G(F)$ such that $H g H$ is closed. Let $x=s(g)$. Then $x$ is a semisimple element of $G$.

For the proof, see e.g. AG2, Proposition 7.2.1.

Definition 2.2.9. In the notation of the previous proposition we will say that the pair $\left(G_{x}, H_{x},\left.\theta\right|_{G_{x}}\right)$ is a descendant of $(G, H, \theta)$.

\subsubsection{Tame symmetric pairs.}

Definition 2.2.10. Let $\pi$ be an action of a reductive group $G$ on a smooth affine variety $X$. We say that an algebraic automorphism $\tau$ of $X$ is $G$-admissible if

(i) $\pi(G(F))$ is of index at most 2 in the group of automorphisms of $X$ generated by $\pi(G(F))$ and $\tau$.

(ii) For any closed $G(F)$ orbit $O \subset X(F)$, we have $\tau(O)=O$.

Definition 2.2.11. We call an action of a reductive group $G$ on a smooth affine variety $X$ tame if for any $G$-admissible $\tau: X \rightarrow X$, every $G(F)$-invariant distribution on $X(F)$ is $\tau$-invariant.

We call a symmetric pair $(G, H, \theta)$ tame if the action of $H \times H$ on $G$ is tame.

Remark 2.2.12. Evidently, any good tame symmetric pair is a GK pair.

Notation 2.2.13. Let $V$ be an algebraic finite dimensional representation over $F$ of a reductive group $G$. Denote $Q(V):=V / V^{G}$. Since $G$ is reductive, there is a canonical embedding $Q(V) \hookrightarrow V$.

Notation 2.2.14. Let $(G, H, \theta)$ be a symmetric pair. We denote by $\mathcal{N}_{G, H}$ the subset of all the nilpotent elements in $Q\left(\mathfrak{g}^{\sigma}\right)$. Denote $R_{G, H}:=Q\left(\mathfrak{g}^{\sigma}\right)-\mathcal{N}_{G, H}$.

Note that our notion of $R_{G, H}$ coincides with the notion $R\left(\mathfrak{g}^{\sigma}\right)$ used in [AG2], Notation 2.3.10. This follows from Lemma 7.1.11 in AG2. 
Definition 2.2.15. We call a symmetric pair $(G, H, \theta)$ weakly linearly tame if for any $H$-admissible transformation $\tau$ of $\mathfrak{g}^{\sigma}$ such that every $H(F)$-invariant distribution on $R_{G, H}$ is also $\tau$-invariant, we have

$\left.{ }^{*}\right)$ every $H(F)$-invariant distribution on $Q\left(\mathfrak{g}^{\sigma}\right)$ is also $\tau$-invariant.

Theorem 2.2.16. Let $(G, H, \theta)$ be a symmetric pair. Suppose that all its descendants (including itself) are weakly linearly tame. Then $(G, H, \theta)$ is tame.

For the proof, see Theorem 7.3.3 in [AG2].

Now we would like to formulate a criterion for being weakly linearly tame. For it we will need the following lemma and notation.

Lemma 2.2.17. Let $(G, H, \theta)$ be a symmetric pair. Then any nilpotent element $x \in \mathfrak{g}^{\sigma}$ can be extended to an sl $l_{2}$ triple $\left(x, d(x), x_{-}\right)$such that $d(x) \in \mathfrak{h}$ and $x_{-} \in \mathfrak{g}^{\sigma}$.

For the proof, see e.g. [AG2, Lemma 7.1.11.

Notation 2.2.18. We will use the notation $d(x)$ from the last lemma in the future. It is not uniquely defined, but whenever we will use this notation nothing will depend on its choice.

Proposition 2.2.19. Let $(G, H, \theta)$ be a symmetric pair. Suppose that for any nilpotent $x \in \mathfrak{g}^{\sigma}$ we have

$$
\operatorname{Tr}\left(\left.\operatorname{ad}(d(x))\right|_{\mathfrak{h}_{x}}\right)<\operatorname{dim} Q\left(\mathfrak{g}^{\sigma}\right) .
$$

Then the pair $(G, H, \theta)$ is weakly linearly tame.

This proposition follows from AG2 (Propositions 7.3.7 and 7.3.5).

\subsubsection{Regular symmetric pairs.}

Definition 2.2.20. Let $(G, H, \theta)$ be a symmetric pair. We call an element $g \in$ $G(F)$ admissible if

(i) $\operatorname{Ad}(g)$ commutes with $\theta$ (or, equivalently, $s(g) \in Z(G)$ ) and

(ii) $\left.A d(g)\right|_{\mathfrak{g}^{\sigma}}$ is $H$-admissible.

Definition 2.2.21. We call a symmetric pair $(G, H, \theta)$ regular if for any admissible $g \in G(F)$ such that every $H(F)$-invariant distribution on $R_{G, H}$ is also $A d(g)$ invariant, we have

$\left(^{*}\right)$ every $H(F)$-invariant distribution on $Q\left(\mathfrak{g}^{\sigma}\right)$ is also $A d(g)$-invariant.

The following two propositions are evident.

Proposition 2.2.22. Let $(G, H, \theta)$ be a symmetric pair. Suppose that any $g \in$ $G(F)$ satisfying $\sigma(g) g \in Z(G(F))$ lies in $Z(G(F)) H(F)$. Then $(G, H, \theta)$ is regular. In particular if the normalizer of $H(F)$ lies inside $Z(G(F)) H(F)$, then $(G, H, \theta)$ is regular.

\section{Proposition 2.2.23.}

(i) Any weakly linearly tame pair is regular.

(ii) A product of regular pairs is regular (see [AG2], Proposition 7.4.4).

In section 4 we will introduce terminology that will help to verify the condition of Proposition 2.2.19.

The importance of the notion of a regular pair is demonstrated by the following theorem. 
Theorem 2.2.24. Let $(G, H, \theta)$ be a good symmetric pair such that all its descendants (including itself) are regular. Then it is a GK pair.

For the proof, see AG2, Theorem 7.4.5.

\section{MAIN RESUlts}

Here we formulate the main results of the paper and explain how they follow from the rest of the paper.

Definition 3.0.1. A quadratic space is a linear space with a fixed nondegenerate quadratic form.

Let $F^{\prime}$ be an extension of $F$ and $V$ be a quadratic space over it. We denote by $O(V)$ the canonical algebraic group such that its $F$-points form the group of orthogonal transformations of $V$.

Definition 3.0.2. Let $D$ be a field with an involution $\tau$. A Hermitian space over $(D, \tau)$ is a linear space over $D$ with a fixed nondegenerate Hermitian form.

Suppose that $D$ is an extension of $F$ and $F \subset D^{\tau}$. Let $V$ be a Hermitian space over $(D, \tau)$. We denote by $U(V)$ the canonical algebraic group such that its $F$-points form the group of unitary transformations of $V$.

Definition 3.0.3. Let $G$ be a reductive group and $\varepsilon \in G$ be an element of order 2 . We denote by $\left(G, G_{\varepsilon}\right)$ the symmetric pair defined by the involution $x \mapsto \varepsilon x \varepsilon$.

The following lemma is straightforward.

Lemma 3.0.4. Let $V$ be a quadratic space.

(i) Let $\varepsilon \in G L(V)$ be an element of order 2. Then $G L(V)_{\varepsilon} \cong G L\left(V_{1}\right) \times G L\left(V_{2}\right)$ for some decomposition $V=V_{1} \oplus V_{2}$.

(ii) Let $\varepsilon \in O(V)$ be an element of order 2. Then $O(V)_{\varepsilon} \cong O\left(V_{1}\right) \times O\left(V_{2}\right)$ for some orthogonal decomposition $V=V_{1} \oplus V_{2}$.

(iii) Let $V$ be a Hermitian space.

Let $\varepsilon \in U(V)$ be an element of order 2. Then $U(V)_{\varepsilon} \cong U\left(V_{1}\right) \times U\left(V_{2}\right)$ for some orthogonal decomposition $V=V_{1} \oplus V_{2}$.

Theorem 3.0.5. Let $V$ be a quadratic space over $F$. Then all the descendants of the pair $\left(O(V), O(V)_{\varepsilon}\right)$ are regular.

Proof. By Theorem 6.5.1 below, the descendants of the pair $\left(O(V), O(V)_{\varepsilon}\right)$ are products of pairs of the types $F$.

(i) $(G L(W), O(W))$ for some quadratic space $W$ over some field $F^{\prime}$ that extends

(ii) $\left(U\left(W_{E}\right), O(W)\right)$ for some quadratic space $W$ over some field $F^{\prime}$ that extends $F$, and some quadratic extension $E$ of $F^{\prime}$. Here, $W_{E}:=W \otimes_{F^{\prime}} E$ is the extension of scalars with the corresponding Hermitian structure.

(iii) $\left(O(W), O(W)_{\varepsilon}\right)$ for some quadratic space $W$ over $F$.

The pair (i) is regular by Theorem 5.1.1 below. The pair (ii) is regular by subsection 5.3 below. The pair (iii) is regular by subsection 5.2 below.

Corollary 3.0.6. Suppose that $F=\mathbb{C}$ and let $V$ be a quadratic space over it. Then the pair $\left(O(V), O(V)_{\varepsilon}\right)$ satisfies $\mathrm{GP} 1$. 
Proof. This pair is good by Proposition 2.2.5 and all its descendants are regular. Hence by Theorem 2.2.24 it is a GK pair. Therefore by Theorem 2.1.4 it satisfies GP2. Now, by Proposition 2.1.5, it satisfies GP1.

Theorem 3.0.7. Let $D / F$ be a quadratic extension and $\tau \in G a l(D / F)$ be the nontrivial element. Let $V$ be a Hermitian space over $(D, \tau)$. Then all the descendants of the pair $\left(U(V), U(V)_{\varepsilon}\right)$ are regular.

Proof. By Theorem 6.6 .1 below, the descendants of the pair $\left(U(V), U(V)_{\varepsilon}\right)$ are products of pairs of the types

(a) $(G \times G, \Delta G)$ for some reductive group $G$.

(b) $(G L(W), U(W))$ for some Hermitian space $W$ over some extension $\left(D^{\prime}, \tau^{\prime}\right)$ of $(D, \tau)$.

(c) $\left(G_{E / F}, G\right)$ for some reductive group $G$ and some quadratic extension $E / F$.

(d) $\left(G L(W), G L(W)_{\varepsilon}\right)$, where $W$ is a linear space over $D$ and $\varepsilon \in G L(W)$ is an element of order $\leq 2$.

(e) $\left(U(W), U(W)_{\varepsilon}\right)$, where $W$ is a Hermitian space over $(D, \tau)$.

The pairs (a) and (c) are regular by Theorem 4.2.11 below. The pairs (b) and (e) are regular by subsection 5.3 below. The pair (d) is regular by Theorem 4.2 .12 below.

Theorem 3.0.8. Let $V$ be a quadratic space over $F$. Then all the descendants of the pair $(G L(V), O(V))$ are weakly linearly tame. In particular, this pair is tame.

Proof. By Theorem 6.2.1 below, the descendants of the pair $(G L(V), O(V))$ are products of pairs of the type $(G L(W), O(W))$ for some quadratic space $W$ over some field $F^{\prime}$ that extends $F$. By Theorem 5.1 .1 below, these pairs are weakly linearly tame. Now, the pair $(G L(V), O(V))$ is tame by Theorem 2.2.16

Corollary 3.0.9. Suppose that $F=\mathbb{C}$ and let $V$ be a quadratic space over it. Then the pair $(G L(V), O(V))$ is GP1.

Theorem 3.0.10. Let $D / F$ be a quadratic extension and $\tau \in G a l(D / F)$ be the nontrivial element. Let $V$ be a Hermitian space over $(D, \tau)$. Then all the descendants of the pair $(G L(V), U(V))$ are weakly linearly tame. In particular, this pair is tame.

Proof. By Theorem 6.3.1 below, all the descendants of the pair $(G L(V), U(V))$ are products of pairs of the types

(i) $(G L(W) \times G L(W), \Delta G L(W))$ for some linear space $W$ over some field $D^{\prime}$ that extends $D$.

(ii) $(G L(W), U(W))$ for some Hermitian space $W$ over some $\left(D^{\prime}, \tau^{\prime}\right)$ that extends $(D, \tau)$.

The pair (i) is weakly linearly tame by Theorem 4.2 .11 below and the pair (ii) is weakly linearly tame by subsection 5.3 below. Now, the pair $(G L(V), U(V))$ is tame by Theorem 2.2.16.

Theorem 3.0.11. Let $V$ be a quadratic space over $F$. Let $D / F$ be a quadratic extension and $\tau \in \operatorname{Gal}(D / F)$ be the nontrivial element. Let $V_{D}:=V \otimes_{F} D$ be its extension of scalars with the corresponding Hermitian structure. Then all the descendants of the pair $\left(U\left(V_{D}\right), O(V)\right)$ are weakly linearly tame. In particular, this pair is tame. 
Proof. By Theorem 6.4.1 below, all the descendants of the pair $\left(U\left(V_{D}\right), O(V)\right)$ are products of pairs of the types

(i) $(G L(W), O(W))$ for some quadratic space $W$ over some field $F^{\prime}$ that extends F.

(ii) $\left(U\left(W_{D^{\prime}}\right), O(W)\right)$ for some extension $\left(D^{\prime}, \tau^{\prime}\right)$ of $(D, \tau)$ and some quadratic space $W$ over $D^{\prime \tau^{\prime}}$.

The pair (i) is weakly linearly tame by Theorem 5.1.1 below, and the pair (ii) is weakly linearly tame by subsection 5.3 below. Now, the pair $(G L(V), U(V))$ is tame by Theorem 2.2.16.

\section{4. $\mathbb{Z} / 2 \mathbb{Z}$ GRADED REPRESENTATIONS OF $s l_{2}$ AND THEIR DEFECTS}

In this section we will introduce terminology that will help to verify the condition of Proposition 2.2.19

\subsection{Graded representations of $s l_{2}$.}

Definition 4.1.1. We fix a standard basis $e, h, f$ of $s l_{2}(F)$. We fix a grading on $s l_{2}(F)$ given by $h \in s l_{2}(F)_{0}$ and $e, f \in s l_{2}(F)_{1}$. A graded representation of $s l_{2}$ is a representation of $s l_{2}$ on a graded vector space $V=V_{0} \oplus V_{1}$ such that $s l_{2}(F)_{i}\left(V_{j}\right) \subset V_{i+j}$, where $i, j \in \mathbb{Z} / 2 \mathbb{Z}$.

The following lemma is standard.

\section{Lemma 4.1.2.}

(i) Every graded representation of $s l_{2}$ which is irreducible as a graded representation is irreducible just as a representation.

(ii) Every irreducible representation $V$ of $s_{2}$ admits exactly two gradings. In one, the highest weight vector lies in $V_{0}$ and in the other lies in $V_{1}$.

Definition 4.1.3. We denote by $V_{\lambda}^{w}$ the irreducible graded representation of $s l_{2}$ with highest weight $\lambda$ and highest weight vector of parity $p$ where $w=(-1)^{p}$.

The following lemma is straightforward.

\section{Lemma 4.1.4.}

$$
\begin{aligned}
& \left(V_{\lambda}^{w}\right)^{*}=V_{\lambda}^{w(-1)^{\lambda}}, \\
& V_{\lambda_{1}}^{w_{1}} \otimes V_{\lambda_{2}}^{w_{2}}=\bigoplus_{i=0}^{\min \left(\lambda_{1}, \lambda_{2}\right)} V_{\lambda_{1}+\lambda_{2}-2 i}^{w_{1} w_{2}(-1)^{i}}, \\
& \Lambda^{2}\left(V_{\lambda}^{w}\right)=\bigoplus_{i=0}^{\left\lfloor\frac{\lambda-1}{2}\right\rfloor} V_{2 \lambda-4 i-2}^{-1} .
\end{aligned}
$$

\subsection{Defects.}

Definition 4.2.1. Let $\pi$ be a graded representation of $s l_{2}$. We define the defect of $\pi$ to be

$$
\operatorname{def}(\pi)=\operatorname{Tr}\left(\left.h\right|_{\left(\pi^{e}\right)_{0}}\right)-\operatorname{dim}\left(\pi_{1}\right) .
$$

The following lemma is straightforward. 


\section{Lemma 4.2.2.}

$$
\begin{aligned}
& \operatorname{def}(\pi \oplus \tau)=\operatorname{def}(\pi)+\operatorname{def}(\tau) . \\
& \operatorname{def}\left(V_{\lambda}^{w}\right)=\frac{1}{2}\left(\lambda w+w\left(\frac{1+(-1)^{\lambda}}{2}\right)-1\right)=\frac{1}{2} \begin{cases}\lambda w+w-1 & \lambda \text { is even, } \\
\lambda w-1 & \lambda \text { is odd. }\end{cases}
\end{aligned}
$$

Definition 4.2.3. Let $\mathfrak{g}$ be a $(\mathbb{Z} / 2 \mathbb{Z})$ graded Lie algebra. We say that $\mathfrak{g}$ is of negative defect if for any graded homomorphism $\pi: s l_{2} \rightarrow \mathfrak{g}$, the defect of $\mathfrak{g}$ with respect to the adjoint action of $s l_{2}$ is negative.

We say that $\mathfrak{g}$ is of negative normalized defect if the semi-simple part of $\mathfrak{g}$ (i.e. the quotient of $\mathfrak{g}$ by its center) is of negative defect.

Remark. Clearly, $\mathfrak{g}$ is of negative normalized defect if and only if for any graded homomorphism $\pi: s l_{2} \rightarrow \mathfrak{g}$, the defect of $\mathfrak{g}$ with respect to the adjoint action of $s l_{2}$ is less than minus the dimension of the odd part of the center of $\mathfrak{g}$.

Definition 4.2.4. We say that a symmetric pair $(G, H, \theta)$ is of negative normalized defect if the Lie algebra $\mathfrak{g}$ with the grading defined by $\theta$ is of negative normalized defect.

Lemma 4.2.5. Let $(G, H, \theta)$ be a symmetric pair. Assume that $\mathfrak{g}$ is semi-simple. Then $Q\left(\mathfrak{g}^{\sigma}\right)=\mathfrak{g}^{\sigma}$.

Proof. Assume the contrary: there exists $0 \neq x \in \mathfrak{g}^{\sigma}$ such that $H x=x$. Then $\operatorname{dim}\left(C N_{H x, x}^{\mathfrak{g}^{\sigma}}\right)=\operatorname{dim} \mathfrak{g}^{\sigma}$, hence $C N_{H x, x}^{\mathfrak{g}^{\sigma}}=\mathfrak{g}^{\sigma}$. On the other hand, $C N_{H x, x}^{\mathfrak{g}^{\sigma}} \cong$ $[\mathfrak{h}, x]^{\perp}=\left(\mathfrak{g}^{\sigma}\right)^{x}$ (here $(\cdot)^{\perp}$ means the orthogonal compliment w.r.t. the Killing form). Therefore $\mathfrak{g}^{\sigma}=\left(\mathfrak{g}^{\sigma}\right)^{x}$ and hence $x$ lies in the center of $\mathfrak{g}$, which is impossible.

Proposition 2.2.19 can be rewritten now in the following form.

Theorem 4.2.6. A symmetric pair of negative normalized defect is weakly linearly tame.

Evidently, a product of pairs of negative normalized defect is again of negative defect.

The following lemma is straightforward.

Lemma 4.2.7. Let $(G, H, \theta)$ be a symmetric pair. Let $F^{\prime}$ be any field extending $F$. Let $\left(G_{F^{\prime}}, H_{F^{\prime}}, \theta\right)$ be the extension of $(G, H, \theta)$ to $F^{\prime}$. Suppose that it is of negative normalized defect (as a pair over $\left.F^{\prime}\right)$. Then $(G, H, \theta)$ and $\left(G_{F^{\prime} / F}, H_{F^{\prime} / F}, \theta\right)$ are of negative normalized defect (as pairs over $F$ ).

In AG2 we proved the following (easy) proposition (see AG2, Lemma 7.6.6).

Proposition 4.2.8. Let $\pi$ be a representation of $s l_{2}$. Then $\operatorname{Tr}\left(\left.h\right|_{\left(\pi^{e}\right)}\right)<\operatorname{dim}(\pi)$.

We would like to reformulate it in terms of defects. For this we will need the following notation.

Notation 4.2.9.

(i) Let $\pi$ be a representation of $s l_{2}$. We denote by $\bar{\pi}$ the representation of $s l_{2}$ on the same space defined by $\bar{\pi}(e):=-\pi(e), \bar{\pi}(f):=-\pi(f)$ and $\bar{\pi}(h):=\pi(h)$.

(ii) We define a grading on $\pi \oplus \bar{\pi}$ by the involution $s(v \oplus w):=w \oplus v$.

Proposition 4.2 .8 can be reformulated in the following way. 
Proposition 4.2.10. Let $\pi$ be a representation of $\operatorname{sl}_{2}$. Then $\operatorname{def}(\pi \oplus \bar{\pi})<0$.

In AG2] we also deduced from this proposition the following theorem (see [AG2], 7.6.2).

Theorem 4.2.11. For any reductive group $G$, the pairs $(G \times G, \Delta G)$ and $\left(G_{E / F}, G\right)$ are of negative normalized defect and hence weakly linearly tame. Here $\Delta G$ is the diagonal in $G \times G$.

In [AG2, $\S \S 7.7]$ we proved the following theorem.

Theorem 4.2.12. The pair $(G L(V \oplus V), G L(V) \times G L(V))$ is of negative normalized defect and hence regular.

Note that in the case $\operatorname{dim} V \neq \operatorname{dim} W$ the pair $(G L(V \oplus W), G L(V) \times G L(W))$ is obviously regular by Proposition 2.2.22.

\section{Proof of Regularity and tameness}

5.1. The pair $(G L(V), O(V))$. In this subsection we prove that the pair $(G L(V)$, $O(V))$ is weakly linearly tame. For $\operatorname{dim} V \leq 1$ it is obvious. Hence it is enough to prove the following theorem.

Theorem 5.1.1. Let $V$ be a quadratic space of dimension at least 2. Then the pair $(G L(V), O(V))$ has negative normalized defect.

We will need the following notation.

Notation 5.1.2. Let $\pi$ be a representation of $s l_{2}$. We define a grading on $\pi \otimes \bar{\pi}$ by the involution $s(v \otimes w):=-w \otimes v$.

Theorem 5.1.1 immediately follows from the following one.

Theorem 5.1.3. Let $\pi$ be a representation of $s_{2}$ of dimension at least 2. Then $\operatorname{def}(\pi \otimes \bar{\pi})<-1$.

This theorem in turn follows from the following lemma.

Lemma 5.1.4. Let $V_{\lambda}$ and $V_{\mu}$ be irreducible representations of $s l_{2}$. Then

(i) $\operatorname{def}\left(V_{\lambda} \otimes \overline{V_{\lambda}}\right)=-(\lambda+1)\left(\frac{\lambda}{2}+1\right)$.

(ii) $\operatorname{def}\left(V_{\lambda} \otimes \overline{V_{\mu}} \oplus V_{\mu} \otimes \overline{V_{\lambda}}\right)<0$.

Proof.

(i) Follows from the fact that $V_{\lambda} \otimes \overline{V_{\lambda}}=\bigoplus_{i=0}^{\lambda} V_{2 \lambda-2 i}^{-1}$ and from Lemma 4.2.2,

(ii) Follows from Proposition 4.2.10,

5.2. The pair $\left(O\left(V_{1} \oplus V_{2}\right), O\left(V_{1}\right) \times O\left(V_{2}\right)\right)$. In this subsection we prove that the pair $\left(O\left(V_{1} \oplus V_{2}\right), O\left(V_{1}\right) \times O\left(V_{2}\right)\right)$ is regular. For that it is enough to prove the following theorem.

Theorem 5.2.1. Let $V_{1}$ and $V_{2}$ be quadratic spaces. Assume $\operatorname{dim} V_{1}=\operatorname{dim} V_{2}$. Then the pair $\left(O\left(V_{1} \oplus V_{2}\right), O\left(V_{1}\right) \times O\left(V_{2}\right)\right)$ has negative normalized defect.

This theorem immediately follows from the following one.

Theorem 5.2.2. Let $\pi$ be a (nonzero) graded representation of $s l_{2}$ such that $\operatorname{dim} \pi_{0}=\operatorname{dim} \pi_{1}$ and $\pi \simeq \pi^{*}$. Then $\Lambda^{2}(\pi)$ has negative defect.

For this theorem we will need the following lemma. 
Lemma 5.2.3. Let $V_{\lambda_{1}}^{w_{1}}$ and $V_{\lambda_{2}}^{w_{2}}$ be irreducible graded representations of $s l_{2}$. Then (i) $\operatorname{def}\left(V_{\lambda_{1}}^{w_{1}} \otimes V_{\lambda_{2}}^{w_{2}}\right)$

$$
=-\frac{1}{2} \begin{cases}\min \left(\lambda_{1}, \lambda_{2}\right)+1-\frac{w_{1} w_{2}}{2}\left(\lambda_{1}+\lambda_{2}+1+(-1)^{\min \left(\lambda_{1}, \lambda_{2}\right)}\left(\left|\lambda_{1}-\lambda_{2}\right|-1\right)\right), & \lambda_{1} \neq \lambda_{2} \quad \bmod 2 ; \\ \min \left(\lambda_{1}, \lambda_{2}\right)+1-w_{1} w_{2}\left(\max \left(\lambda_{1}, \lambda_{2}\right)+1\right), & \lambda_{1} \equiv \lambda_{2} \equiv 0 \quad \bmod 2 \\ \min \left(\lambda_{1}, \lambda_{2}\right)+1-w_{1} w_{2}\left(\min \left(\lambda_{1}, \lambda_{2}\right)+1\right), & \lambda_{1} \equiv \lambda_{2} \equiv 1 \quad \bmod 2\end{cases}
$$

(ii) $\operatorname{def}\left(\Lambda^{2}\left(V_{\lambda_{1}}^{w_{1}}\right)\right)=-\frac{\lambda^{2}}{4}-\frac{\lambda}{2}-\frac{1+(-1)^{\lambda+1}}{8}$.

Proof. This lemma follows by straightforward computations from Lemmas 4.1.4 and 4.2 .2 .

Proof of Theorem 5.2.2. Since $\pi \simeq \pi^{*}, \pi$ can be decomposed to a direct sum of irreducible graded representations in the following way:

$$
\pi=\left(\bigoplus_{i=1}^{l} V_{\lambda_{i}}^{1}\right) \oplus\left(\bigoplus_{j=1}^{m} V_{\mu_{j}}^{-1}\right) \oplus\left(\bigoplus_{k=1}^{n} V_{\nu_{k}}^{1} \oplus V_{\nu_{k}}^{-1}\right) .
$$

Here, all $\lambda_{i}$ and $\mu_{j}$ are even and $\nu_{k}$ are odd. Since $\operatorname{dim} \pi_{0}=\operatorname{dim} \pi_{1}, l=m$.

By the last lemma, $\operatorname{def}\left(V_{\lambda_{i}}^{1} \otimes\left(V_{\nu_{k}}^{1} \oplus V_{\nu_{k}}^{-1}\right)\right)=-\left(\min \left(\lambda_{1}, \lambda_{2}\right)+1\right)<0$. Similarly, $\operatorname{def}\left(V_{\mu_{j}}^{-1} \otimes\left(V_{\nu_{k}}^{1} \oplus V_{\nu_{k}}^{-1}\right)\right)<0$. Also, $\operatorname{def}\left(\left(V_{\nu_{k_{1}}}^{1} \oplus V_{\nu_{k_{1}}}^{-1}\right) \otimes\left(V_{\nu_{k_{2}}}^{1} \oplus V_{\nu_{k_{2}}}^{-1}\right)\right)<0$ and $\operatorname{def}\left(\Lambda^{2}\left(V_{\lambda}^{w}\right)\right) \leq 0$ for all $\lambda$ and $w$.

Hence if $l=0$ we are done. Otherwise we can assume $n=0$. Now,

$$
\begin{aligned}
& \operatorname{def}\left(\Lambda^{2}(\pi)\right) \leq \sum_{1 \leq i<j \leq l}\left|\lambda_{i}-\lambda_{j}\right|+\sum_{1 \leq i<j \leq l}\left|\mu_{i}-\mu_{j}\right|-\sum_{1 \leq i, j \leq l}\left(\lambda_{i}+\mu_{j}+2\right) \\
& <\sum_{1 \leq i<j \leq l}\left(\lambda_{i}+\lambda_{j}\right)+\sum_{1 \leq i<j \leq l}\left(\mu_{i}+\mu_{j}\right)-\sum_{1 \leq i, j \leq l}\left(\lambda_{i}+\mu_{j}\right)=-\sum_{i=1}^{l}\left(\lambda_{i}+\mu_{i}\right) \leq 0 .
\end{aligned}
$$

5.3. The pairs $(G L(V), U(V)),\left(U\left(V_{1} \oplus V_{2}\right), U\left(V_{1}\right) \times U\left(V_{2}\right)\right)$ and $\left(U\left(V_{D}\right), O(V)\right)$. In this subsection we prove that the pairs $(G L(V), U(V))$ and $\left(U\left(V_{D}\right), O(V)\right)$ are weakly linearly tame and the pair $\left(U\left(V_{1} \oplus V_{2}\right), U\left(V_{1}\right) \times U\left(V_{2}\right)\right)$ is regular.

Let $V$ be a Hermitian space. Note that $(G L(V), U(V))$ is a form of $(G L(W) \times$ $G L(W), \Delta G L(W))$ for some $W$ and $(U(V \oplus V), U(V) \times U(V))$ is a form of $(G L(W \oplus$ $W), G L(W)) \times G L(W))$ for some $W$.

Also, for any quadratic space $V$ of dimension at least 2 and any quadratic extension $D / F$, the pair $\left(U\left(V_{D}\right), O(V)\right)$ is a form of $(G L(W), O(W))$ for some quadratic space $W$.

Hence by Lemma 4.2.7 and Theorems 4.2.11 4.2.12 and 5.1.1 those 3 pairs are of negative normalized defect and hence are weakly linearly tame. If $\operatorname{dim} V \leq 1$, then the pair $\left(U\left(V_{D}\right), O(V)\right)$ is obviously linearly tame.

If $V_{1}$ and $V_{2}$ are nonisomorphic Hermitian spaces, then $\left(U\left(V_{1} \oplus V_{2}\right), U\left(V_{1}\right) \times\right.$ $\left.U\left(V_{2}\right)\right)$ is regular by Proposition 2.2.22.

\section{Computation of Descendants}

In this section we compute the descendants of the pairs we discussed before. For this we use a technique of computing centralizers of semisimple elements of 
orthogonal and unitary groups, which is described in SpSt. The proofs in this section are rather straightforward but technically involved. The most important things in this section are the formulations of the main theorems: Theorems 6.2.1, 6.3.1, 6.4.1, 6.5.1, 6.6.1. Those theorems are summarized graphically in subsection 6.7 .

\subsection{Preliminaries and notation for orthogonal and unitary groups.}

\subsubsection{Orthogonal group.}

Notation 6.1.1. Let $V$ be a linear space over $F$. Let $x \in G L(V)$ be a semisimple element and let $Q=\sum_{i=0}^{n} a_{i} \xi^{i} \in F[\xi]\left(\right.$ where $\left.a_{n} \neq 0\right)$ be an irreducible polynomial.

- Denote $F_{Q}:=F[\xi] / Q$.

- Denote $i n v(Q):=\sum_{i=0}^{n} a_{n-i} \xi^{i}$.

- Denote $V_{Q, x}^{0}:=\operatorname{Ker}(Q(x))$ and $V_{Q, x}^{1}:=\operatorname{Ker}(\operatorname{inv}(Q)(x))$.

We define an $F_{Q}$-linear space structure on $V_{Q, x}^{i}$ by letting $\xi$ act on $V_{Q, x}^{0}$ by $x$ and on $V_{Q, x}^{1}$ by $x^{-1}$. We will consider $V_{Q, x}^{i}$ as linear spaces over $F_{Q}$.

- In case $Q$ is proportional to $\operatorname{inv}(Q)$, we define an involution $\mu$ on $F_{Q}$ by $\mu(P(\xi)):=P\left(\xi^{-1}\right)$.

- For a linear space $W$ over $F_{Q}$ we can consider its dual space $W^{*}$ over $F_{Q}$ and the dual space of $W$ over $F$ which we denote by $W_{F}^{*}$. The space $W_{F}^{*}$ has a canonical structure of a linear space over $F_{Q}$. The spaces $W_{F}^{*}$ and $W^{*}$ can be identified as linear spaces over $F_{Q}$. For this identification one has to choose an $F$-linear functional $\lambda: F_{Q} \rightarrow F$. We will fix such a functional $\lambda$ such that $\lambda(\mu(d))=\lambda(d)$ if $\mu$ is defined.

From now on we will identify $W_{F}^{*}$ and $W^{*}$.

The following two lemmas are straightforward.

Lemma 6.1.2. Let $V$ be a quadratic space over $F$. Let $x \in G L(V)$ and let $P, Q \in$ $F[\xi]$ be irreducible polynomials. Suppose that either

(i) $x=x^{t}$ and $P$ is not proportional to $Q$ or

(ii) $x \in O(V)$ and $P$ is not proportional to inv $(Q)$.

Then $\operatorname{Ker}(Q(x))$ is orthogonal to $\operatorname{Ker}(P(x))$.

Lemma 6.1.3. Let $(V, B)$ be a quadratic space over $F$. Let $x \in G L(V)$ be a semisimple element and let $Q \in F[\xi]$ be an irreducible polynomial. Then

(i) if $x=x^{t}$, then $B$ defines an $F_{Q}$-linear isomorphism $V_{Q, x}^{i} \cong\left(V_{Q, x}^{i}\right)^{*}$.

(ii) if $x \in O(V)$, then $B$ defines an $F_{Q}$-linear isomorphism $V_{Q, x}^{i} \cong\left(V_{Q, x}^{1-i}\right)^{*}$.

6.1.2. Unitary group. From now and until the end of the paper we fix a quadratic extension $D$ of $F$ and denote by $\tau$ the involution that fixes $F$.

Notation 6.1.4. Let $V$ be a Hermitian space over $(D, \tau)$. Let $x \in G L(V)$ be a semisimple element and let $Q=\sum_{i=0}^{n} a_{i} \xi^{i} \in D[\xi]$ (where $a_{n} \neq 0$ ) be an irreducible polynomial.

- Denote $D_{Q}:=D[\xi] / Q$.

- Denote

$$
\operatorname{inv}(Q):=\sum_{i=0}^{n} a_{n-i} \xi^{i}, \quad Q^{*}:=\tau(\operatorname{inv}(Q)) .
$$


- Denote

$$
\begin{gathered}
V_{Q, x}^{00}:=\operatorname{Ker}(Q(x)), \quad V_{Q, x}^{01}:=\operatorname{Ker}\left(Q^{*}(x)\right), \\
V_{Q, x}^{10}:=\operatorname{Ker}(\operatorname{inv}(Q)(x)), \quad V_{Q, x}^{11}:=\operatorname{Ker}(\tau(Q)(x)) .
\end{gathered}
$$

We twist the action of $D$ on $V_{Q, x}^{i 1}$ by $\tau$. We define a $D_{Q}$-linear space structure on $V_{Q, x}^{i j}$ by letting $\xi$ act on $V_{Q, x}^{0 j}$ by $x$ and on $V_{Q, x}^{1 j}$ by $x^{-1}$. We will consider $V_{Q, x}^{i j}$ as linear spaces over $D_{Q}$.

- If $Q$ is proportional to $Q^{*}$ we define an involution $\mu^{01}$ on $D_{Q}$ by $\mu^{01}(P(\xi)):=$ $\tau(P)\left(\xi^{-1}\right)$.

If $Q$ is proportional to $\operatorname{inv}(Q)$ we define an involution $\mu^{10}$ on $D_{Q}$ by $\mu^{10}(P(\xi)):=P\left(\xi^{-1}\right)$.

If $Q$ is proportional to $\tau(Q)$ we define an involution $\mu^{11}$ on $D_{Q}$ by $\mu^{11}(P(\xi))$ $:=\tau(P)(\xi)$.

- For a linear space $W$ over $D_{Q}$ we can consider its dual space $W^{*}$ over $D_{Q}$ and the dual space of $W$ over $D$, which we denote by $W_{D}^{*}$. The space $W_{D}^{*}$ has a canonical structure of a linear space over $D_{Q}$. The spaces $W_{D}^{*}$ and $W^{*}$ can be identified as linear spaces over $D_{Q}$. For this identification one has to choose a $D$-linear functional $\lambda: D_{Q} \rightarrow D$. We will fix such a functional $\lambda$ such that

$$
\lambda\left(\mu^{i j}(d)\right)=\tau^{j}(\lambda(d)) \quad \text { if } \mu^{i j} \text { is defined. }
$$

From now on we will identify $W_{D}^{*}$ and $W^{*}$.

The following two lemmas are straightforward.

Lemma 6.1.5. Let $V$ be a Hermitian space over $(D, \tau)$. Let $x \in G L(V)$ and let $P, Q \in D[\xi]$ be irreducible polynomials. Suppose that either

(i) $x=x^{*}$ and $P$ is not proportional to $\tau(Q)$ or

(ii) $x \in U(V)$ and $P$ is not proportional to $Q^{*}$.

Then $\operatorname{Ker}(Q(x))$ is orthogonal to $\operatorname{Ker}(P(x))$.

Lemma 6.1.6. Let $(V, B)$ be a Hermitian space over $(D, \tau)$. Let $x \in G L(V)$ be a semisimple element and let $Q \in D[\xi]$ be an irreducible polynomial. Then

(i) If $x=x^{*}$, then $B$ defines a $D_{Q}$-linear isomorphism $V_{Q, x}^{i j} \cong\left(V_{Q, x}^{1-i, 1-j}\right)^{*}$.

(ii) If $x \in U(V)$, then $B$ defines a $D_{Q}$-linear isomorphism $V_{Q, x}^{i j} \cong\left(V_{Q, x}^{i, 1-j}\right)^{*}$.

6.2. The pair $(G L(V), O(V))$.

Theorem 6.2.1. Let $V$ be a quadratic space over $F$. Then all the descendants of the pair $(G L(V), O(V))$ are products of pairs of the type $(G L(W), O(W))$ for some quadratic space $W$ over some field $F^{\prime}$ that extends $F$.

Proof. Note that in this case the anti-involution $\sigma$ is given by $\sigma(x)=x^{t}$. Let $x \in G L(V)^{\sigma}$ be a semisimple element. Let $P$ be the minimal polynomial of $x$. We will now discuss a special case and then deduce the general case from it.

Case 1. $P$ is irreducible over $F$.

Clearly $G L(V)_{x} \cong G L\left(V_{P, x}^{0}\right)$. The isomorphism $V_{P, x}^{0} \cong\left(V_{P, x}^{0}\right)^{*}$ gives a quadratic structure on $V_{P, x}^{0}$. Now $O(V)_{x} \cong O\left(V_{P, x}^{0}\right)$. 
Case 2. General case.

Let $P=\prod_{i \in I} P_{i}$ be the decomposition of $P$ to irreducible polynomials. Clearly $V=\bigoplus V_{P_{i}, x}^{0}$ and $V_{P_{i}, x}^{0}$ are orthogonal to each other. Hence the pair $\left(G L(V)_{x}\right.$, $\left.O(V)_{x}\right)$ is a product of pairs from Case 1.

\subsection{The pair $(G L(V), U(V))$.}

Theorem 6.3.1. Let $(V, B)$ be a Hermitian space over $(D, \tau)$. Then all the descendants of the pair $(G L(V), U(V))$ are products of pairs of the types

(i) $(G L(W) \times G L(W), \Delta G L(W))$ for some linear space $W$ over some field $D^{\prime}$ that extends $D$;

(ii) $(G L(W), U(W))$ for some Hermitian space $W$ over some $\left(D^{\prime}, \tau^{\prime}\right)$ that extends $(D, \tau)$.

Proof. Note that in this case the anti-involution $\sigma$ is given by $\sigma(x)=x^{*}$. Let $x \in G L(V)^{\sigma}$ be a semisimple element. Let $P$ be the minimal polynomial of $x$. Note that $\tau(P)$ is proportional to $P$. We will now discuss 2 special cases and then deduce the general case from them.

Case 1. $P=Q \tau(Q)$, where $Q$ is irreducible over $D$.

Clearly $G L(V)_{x} \cong G L\left(V_{Q, x}^{00}\right) \times G L\left(V_{Q, x}^{11}\right)$. Recall that $B$ gives a nondegenerate pairing between $V_{Q, x}^{00}$ and $V_{Q, x}^{11}$, and the spaces $V_{Q, x}^{i i}$ are isotropic. Therefore

and

$$
G L\left(V_{Q, x}^{00}\right) \cong G L\left(V_{Q, x}^{11}\right), \quad G L(V)_{x} \cong G L\left(V_{Q, x}^{00}\right)^{2}
$$

$$
U(V)_{x} \cong \Delta G L\left(V_{Q, x}^{00}\right)<G L\left(V_{Q, x}^{00}\right)^{2} .
$$

Case 2. $P$ is irreducible over $D$.

Clearly $G L(V)_{x} \cong G L\left(V_{P, x}^{00}\right)$ and $V_{P, x}^{00}$ is identical to $V_{P, x}^{11}$ as $F$-linear spaces, but the actions of $D_{P}$ differ by a twist by $\mu^{11}$. Hence the isomorphism $V_{P, x}^{00} \cong\left(V_{P, x}^{11}\right)^{*}$ gives a Hermitian structure on $V_{P, x}^{00}$ over $\left(D_{P}, \mu^{11}\right)$. Now $U(V)_{x} \cong U\left(V_{P, x}^{00}\right)<$ $G L\left(V_{P, x}^{00}\right)$.

Case 3. General case.

Let $P=\prod_{i \in I} P_{i}$ be the decomposition of $P$ to irreducible polynomials. Then $\tau\left(P_{i}\right)$ is proportional to $P_{s(i)}$, where $s$ is some permutation of $I$ of order $\leq 2$. Let $I=\bigsqcup I_{\alpha}$ be the decomposition of $I$ to orbits of $s$. Denote $V_{\alpha}:=\operatorname{Ker}\left(\prod_{i \in \alpha} P_{i}(x)\right)$. Clearly $V=\bigoplus V_{\alpha}$ and $V_{\alpha}$ are orthogonal to each other. Hence the pair $\left(G L(V)_{x}\right.$, $\left.U(V)_{x}\right)$ is a product of pairs from the first 2 cases.

\subsection{The pair $\left(U\left(V_{D}\right), O(V)\right)$.}

Theorem 6.4.1. Let $(V, B)$ be a quadratic space over $F$. Let $V_{D}:=V \otimes_{F} D$ be its extension of scalars with the corresponding Hermitian structure.

Then all the descendants of the pair $\left(U\left(V_{D}\right), O(V)\right)$ are products of pairs of the types

(i) $(G L(W), O(W))$ for some quadratic space $W$ over some field $F^{\prime}$ that extends $F$.

(ii) $\left(U\left(W_{D^{\prime}}\right), O(W)\right)$ for some extension $\left(D^{\prime}, \tau^{\prime}\right)$ of $(D, \tau)$ and some quadratic space $W$ over $D^{\prime \tau^{\prime}}$. 
For the proof of this theorem we will need the following notation and lemma.

Notation 6.4.2. Let $(V, B)$ be a quadratic space over $F$. The involution $\tau$ defines an involution $\widetilde{\tau}$ on $V_{D}$. The form $B$ defines a quadratic form $B_{D}$ on $V_{D}$ and a Hermitian form $B_{D}^{\tau}$ on $V_{D}$.

Lemma 6.4.3. Let $(V, B)$ be a quadratic space over $F$. Let $P$ be an irreducible polynomial. Let $x \in U\left(V_{D}\right)$ be a semisimple element such that $x=x^{t}$ (where $x^{t}$ is defined by $\left.B_{D}\right)$. Then the involution $\widetilde{\tau}$ gives a $D_{P}$-linear isomorphism $V_{P, x}^{i j} \cong$ $V_{P, x}^{i, 1-j}$.

Proof. We will show that $\widetilde{\tau}$ maps $V_{P, x}^{00}$ to $V_{P, x}^{11}$, and the other cases are done similarly. Let $v \in V_{P, x}^{i j}$. We have

$$
P^{*}(x)(\widetilde{\tau}(v))=\widetilde{\tau}\left(\operatorname{inv}(P)\left(x^{*}\right)(v)\right)=\widetilde{\tau}\left(\operatorname{inv}(P)\left(x^{-1}\right)(v)\right)=\widetilde{\tau}\left(x^{-\operatorname{deg} P} P(x)(v)\right)=0 .
$$

Proof of Theorem 6.4.1. Note that in this case the anti-involution $\sigma$ is given by $\sigma(x)=x^{t}$. Let $x \in U\left(V_{D}\right)^{\sigma}$ be a semisimple element. Let $P$ be the minimal polynomial of $x$. Then $P$ is proportional to $P^{*}$. We will now discuss 2 special cases and then deduce the general case from them.

Case 1. $P=Q Q^{*}$, where $Q$ is irreducible over $D$.

Clearly $G L(V)_{x} \cong G L\left(V_{Q, x}^{00}\right) \times G L\left(V_{Q, x}^{01}\right)$. Recall that $B_{D}^{\tau}$ gives a nondegenerate pairing between $V_{Q, x}^{00}$ and $V_{Q, x}^{01}$, and the spaces $V_{Q, x}^{0 i}$ are isotropic. Therefore

$$
\begin{gathered}
G L\left(V_{Q, x}^{00}\right) \cong G L\left(V_{Q, x}^{01}\right), \quad G L(V)_{x} \cong G L\left(V_{Q, x}^{00}\right)^{2}, \\
U(V)_{x} \cong \Delta G L\left(V_{Q, x}^{00}\right)<G L\left(V_{Q, x}^{00}\right)^{2} .
\end{gathered}
$$

Compose the isomorphism $V_{Q, x}^{00} \cong V_{Q, x}^{01}$ given by $\widetilde{\tau}$ with the isomorphism $V_{Q, x}^{01} \cong$ $\left(V_{Q, x}^{00}\right)^{*}$ given by $B_{D}^{\tau}$. This gives a quadratic structure on $V_{Q, x}^{00}$. Now

$$
O(V)_{x} \cong \Delta O\left(V_{Q, x}^{00}\right)<\Delta G L\left(V_{Q, x}^{00}\right) .
$$

Case 2. $P$ is irreducible over $D$.

Clearly $G L(V)_{x} \cong G L\left(V_{P, x}^{00}\right)$ and $V_{P, x}^{00}$ is identical to $V_{P, x}^{01}$ as $F$-linear spaces, but the actions of $D_{P}$ on them differ by a twist by $\mu^{01}$. Hence the isomorphism $V_{P, x}^{00} \cong\left(V_{P, x}^{01}\right)^{*}$ given by $B_{D}^{\tau}$ gives a Hermitian structure on $V_{P, x}^{00}$ over $\left(D_{P}, \mu^{01}\right)$ and the isomorphism $V_{P, x}^{00} \cong V_{P, x}^{01}$ given by $\widetilde{\tau}$ gives an antilinear involution of $V_{P, x}^{00}$. Now

$$
U(V)_{x} \cong U\left(V_{P, x}^{00}\right)<G L\left(V_{P, x}^{00}\right) \text { and } O(V)_{x} \cong O\left(V_{P, x}^{00}\right)<U\left(V_{P, x}^{00}\right) .
$$

Case 3. General case.

Let $P=\prod_{i \in I} P_{i}$ be the decomposition of $P$ to irreducible polynomials. Then $P_{i}^{*}$ is proportional to $P_{s(i)}$, where $s$ is some permutation of $I$ of order $\leq 2$. Let $I=\bigsqcup I_{\alpha}$ be the decomposition of $I$ to orbits of $s$. Denote $V_{\alpha}:=\operatorname{Ker}\left(\prod_{i \in \alpha} P_{i}(x)\right)$. Clearly $V_{D}=\bigoplus V_{\alpha}, V_{\alpha}$ are orthogonal to each other and each $V_{\alpha}$ is invariant with respect to $\widetilde{\tau}$. Hence the pair $\left(G L(V)_{x}, U(V)_{x}\right)$ is a product of pairs from the first 2 cases. 
6.5. The pair $\left(O\left(V_{1} \oplus V_{2}\right), O\left(V_{1}\right) \times O\left(V_{2}\right)\right)$.

Theorem 6.5.1. Let $(V, B)$ be a quadratic space over $F$.

Let $\varepsilon \in O(V)$ be an element of order 2. Then all the descendants of the pair $\left(O(V), O(V)_{\varepsilon}\right)$ are products of pairs of the types F.

(i) $(G L(W), O(W))$ for some quadratic space $W$ over some field $F^{\prime}$ that extends

(ii) $\left(U\left(W_{E}\right), O(W)\right)$ for some quadratic space $W$ over some field $F^{\prime}$ that extends $F$, and some quadratic extension $E$ of $F^{\prime}$.

(iii) $\left(O(W), O(W)_{\varepsilon}\right)$ for some quadratic space $W$ over $F$.

For the proof of this theorem we will need the following straightforward lemma.

Lemma 6.5.2. Let $(V, B)$ be a quadratic space over $F$.

Let $\varepsilon \in O(V)$ be an element of order 2. Let $x \in O(V)$ such that $\varepsilon x \varepsilon=x^{-1}$. Let $Q$ be an irreducible polynomial. Then $\varepsilon$ gives an $F_{Q}$ - linear isomorphism $V_{Q, x}^{i} \cong V_{Q, x}^{1-i}$.

Proof of Theorem 6.5.1. Note that the involution $\sigma$ on $O(V)$ is given by $x \mapsto \varepsilon x^{-1} \varepsilon$. Let $x \in O(V)^{\sigma}$ be a semisimple element and let $P$ be its minimal polynomial.

Note that the minimal polynomial of $x^{-1}$ is $\operatorname{inv}(P)$, and hence $P$ is proportional to $\operatorname{inv}(P)$. We will now discuss 3 special cases and then deduce the general case from them.

Case 1. $P=\operatorname{Qinv}(Q)$, where $Q$ is an irreducible polynomial.

Note that $G L(V)_{x} \cong \prod_{i} G L\left(V_{Q, x}^{i}\right)$.

Since $B$ defines a nondegenerate pairing $V_{Q, x}^{0} \cong\left(V_{Q, x}^{1}\right)^{*}$, and $V_{Q, x}^{i}$ are isotropic, we have

$$
O(V)_{x} \cong \Delta G L\left(V_{Q, x}^{0}\right)<G L\left(V_{Q, x}^{0}\right)^{2} .
$$

Now, compose the isomorphism $V_{Q, x}^{i} \cong V_{Q, x}^{1-i}$ given by $\varepsilon$ with the isomorphism $V_{Q, x}^{1-i} \cong\left(V_{Q, x}^{i}\right)^{*}$. This gives a quadratic structure on $V_{Q, x}^{0}$. Clearly, $\varepsilon$ gives an isomorphism $V_{Q, x}^{0} \cong V_{Q, x}^{1}$ as quadratic spaces and hence

$$
\left(O(V)_{\varepsilon}\right)_{x} \cong \Delta O\left(V_{Q, x}^{0}\right)<\Delta G L\left(V_{Q, x}^{0}\right) .
$$

Case 2. $P$ is irreducible and $x \neq x^{-1}$.

In this case $G L(V)_{x} \cong G L\left(V_{P, x}^{0}\right)$. Also, $V_{P, x}^{0}$ and $V_{P, x}^{1}$ are identical as $F$-vector spaces, but the action of $F_{P}$ on them differs by a twist by $\mu$. Therefore the isomorphism $V_{P, x}^{0} \cong\left(V_{P, x}^{1}\right)^{*}$ gives a Hermitian structure on $V_{P, x}^{0}$ over $\left(F_{P}, \mu\right)$ and $\varepsilon$ gives an $\left(F_{P}, \mu\right)$-antilinear automorphism of $V_{P, x}^{0}$. Now

$$
O(V)_{x} \cong U\left(V_{P, x}^{0}\right) .
$$

Denote $W:=\left(V_{P, x}^{0}\right)^{\varepsilon}$. It is a linear space over $\left(F_{P}\right)^{\mu}$. It has a quadratic structure. Now

$$
\left(O(V)_{\varepsilon}\right)_{x} \cong O(W)<U\left(V_{P, x}^{0}\right) .
$$

Case 3. $P$ is irreducible and $x=x^{-1}$.

Again, $G L(V)_{x} \cong G L\left(V_{P, x}^{0}\right)$. However, in this case, $F_{P}=F$ and $V_{P, x}^{0}=V$. Also $O(V)_{x} \cong O\left(V_{P, x}^{0}\right)$. Now, $\varepsilon$ commutes with $x$ and hence $\varepsilon \in O(V)_{x} \cong O\left(V_{P, x}^{0}\right)$. Hence

$$
\left(O(V)_{\varepsilon}\right)_{x} \cong\left(O\left(V_{P, x}^{0}\right)\right)_{\varepsilon}<O\left(V_{P, x}^{0}\right) .
$$


Case 4. General case.

Let $P=\prod_{i \in I} P_{i}$ be the decomposition of $P$ to irreducible multiples. Since $P$ is proportional to $\operatorname{inv}(P)$, every $P_{i}$ is proportional to $P_{s(i)}$ where $s$ is some permutation of $I$ of order $\leq 2$.

Let $I=\bigsqcup I_{\alpha}$ be the decomposition of $I$ to the orbits of $s$. Denote $V_{\alpha}:=$ $\operatorname{Ker}\left(\prod_{i \in \alpha} P_{i}(x)\right)$. Clearly $V=\bigoplus V_{\alpha}$ and the $V_{\alpha}$ are orthogonal to each other and $\varepsilon$-invariant. Hence the pair $\left(O(V)_{x},\left(O(V)_{\varepsilon}\right)_{x}\right)$ is a product of pairs from the first 3 cases.

6.6. The pair $\left(U\left(V_{1} \oplus V_{2}\right), U\left(V_{1}\right) \times U\left(V_{2}\right)\right)$.

Theorem 6.6.1. Let $(V, B)$ be a Hermitian space over $(D, \tau)$.

Let $\varepsilon \in U(V)$ be an element of order 2. Then all the descendants of the pair $\left(U(V), U(V)_{\varepsilon}\right)$ are products of pairs of the types

(i) $(G L(W) \times G L(W), \Delta G L(W))$ for some linear space $W$ over some field $D^{\prime}$ that extends $D$.

(ii) $(U(W) \times U(W), \Delta U(W))$ for some Hermitian space $W$ over some extension $\left(D^{\prime}, \tau^{\prime}\right)$ of $(D, \tau)$.

(iii) $(G L(W), U(W))$ for some Hermitian space $W$ over some extension $\left(D^{\prime}, \tau^{\prime}\right)$ of $(D, \tau)$.

(iv) $\left(G L\left(W_{D^{\prime}}\right), G L(W)\right)$, where $F^{\prime}$ is a field extension of $D, D^{\prime} / F^{\prime}$ is a quadratic extension, $W$ is a linear space over $F^{\prime}$ and $W_{D^{\prime}}:=W \otimes_{F^{\prime}} D^{\prime}$ is its extension of scalars to $D^{\prime}$.

(v) $\left(G L(W), G L(W)_{\varepsilon}\right)$, where $W$ is a linear space over $D$ and $\varepsilon \in G L(W)$ is an element of order $\leq 2$.

(vi) $\left(U\left(W_{E}\right), \bar{U}(W)\right)$, where $W$ is a Hermitian space over some extension $\left(D^{\prime}, \tau^{\prime}\right)$ of $(D, \tau),\left(E, \tau^{\prime \prime}\right)$ is some quadratic extension of $\left(D^{\prime}, \tau^{\prime}\right)$ and $W_{E}=W \otimes_{D^{\prime}} E$ is an extension of scalars with the corresponding Hermitian structure.

(vii) $\left(U(W), U(W)_{\varepsilon}\right)$, where $W$ is a Hermitian space over $(D, \tau)$.

For the proof of this theorem we will need the following straightforward lemma.

Lemma 6.6.2. Let $(V, B)$ be a Hermitian space over $(D, \tau)$. Let $\varepsilon \in U(V)$ be an element of order 2. Let $x \in U(V)$ such that $\varepsilon x \varepsilon=x^{-1}$. Let $Q$ be an irreducible polynomial. Then $\varepsilon$ gives a $D_{Q}$ - linear isomorphism $V_{Q, x}^{i j} \cong V_{Q, x}^{1-i, j}$.

Proof of Theorem 6.6.1. Let $x \in U(V)^{\sigma}$ be a semisimple element and let $P$ be its minimal polynomial.

Note that the minimal polynomial of $x^{*}$ is $P^{*}$, and hence $P^{*}$ is proportional to $P$. Since $x \in U(V)^{\sigma}$, we have $x^{-1}=\varepsilon x \varepsilon$ and hence its minimal polynomial is $P$. Hence $P$ is proportional to $\operatorname{inv}(P)$. We will now discuss 7 special cases and then deduce the general case from them.

Case 1. $P=Q Q^{*} \operatorname{inv}(Q) \tau(Q)$, where $Q$ is an irreducible polynomial.

Note that $G L(V)_{x} \cong \prod_{i j} G L\left(V_{Q, x}^{i j}\right) \cong G L\left(V_{Q, x}^{00}\right)^{4}$. This identifies $U(V)_{x}$ with a diagonal $\Delta G L\left(V_{Q, x}^{00}\right)^{2}<G L\left(V_{Q, x}^{00}\right)^{4}$ and $\left(U(V)_{\varepsilon}\right)_{x}$ with a diagonal $\Delta G L\left(V_{Q, x}^{00}\right)<$ $G L\left(V_{Q, x}^{00}\right)^{4}$.

Case 2. $P=Q \operatorname{Rinv}(Q)$, where $Q$ is an irreducible polynomial and $Q^{*}=Q$.

Note that $G L(V)_{x} \cong \prod_{i} G L\left(V_{Q, x}^{i 0}\right) \cong G L\left(V_{Q, x}^{00}\right)^{2}$. Note also that in this case $V_{Q, x}^{i 0}$ and $V_{Q, x}^{i 1}$ are identical as sets and $F$-vector spaces but the actions of $D_{Q}$ 
on them differ by a twist by $\mu^{01}$. Now the isomorphism $V_{Q, x}^{i 0} \cong\left(V_{Q, x}^{i 1}\right)^{*}$ gives a $\left(D_{Q}, \mu^{01}\right)$-Hermitian structure on $V_{Q, x}^{i 0}$. Therefore, $U(V)_{x} \cong U\left(V_{Q, x}^{00}\right) \times U\left(V_{Q, x}^{10}\right)$. Note that $\varepsilon$ gives an isomorphism of $\left(D_{Q}, \mu^{01}\right)$-Hermitian spaces between $V_{Q, x}^{00}$ and $V_{Q, x}^{01}$. Hence

$$
U(V)_{x} \cong U\left(V_{Q, x}^{00}\right)^{2} \text { and }\left(U(V)_{\varepsilon}\right)_{x} \cong \Delta U\left(V_{Q, x}^{00}\right)<U\left(V_{Q, x}^{00}\right)^{2} .
$$

Case 3. $P=\operatorname{Qinv}(Q)$, where $Q$ is an irreducible polynomial and $Q^{*}=i n v(Q)$.

Note that $G L(V)_{x} \cong \prod_{i} G L\left(V_{Q, x}^{i 0}\right) \cong \prod_{j} G L\left(V_{Q, x}^{0 j}\right)$.

Since $B$ defines a nondegenerate pairing $V_{Q, x}^{00} \cong\left(V_{Q, x}^{01}\right)^{*}$ and $V_{Q, x}^{0 i}$ are isotropic, we have

$$
U(V)_{x} \cong \Delta G L\left(V_{Q, x}^{00}\right)<\left(G L\left(V_{Q, x}^{00}\right)\right)^{2} .
$$

Note that in this case $V_{Q, x}^{i j}$ and $V_{Q, x}^{1-i, 1-j}$ are identical as sets and as $F$-vector spaces, but the action of $D_{Q}$ on them differs by a twist by $\mu^{11}$.

Now, compose the isomorphism $V_{Q, x}^{00} \cong V_{Q, x}^{10}$ given by $\varepsilon$ with the isomorphism $V_{Q, x}^{10} \cong\left(V_{Q, x}^{11}\right)^{*}$. This gives a $\left(D_{Q}, \mu^{11}\right)$ unitary structure on $V_{Q, x}^{00}$. Similarly we get a unitary structure on $V_{Q, x}^{10}$. Finally, $\varepsilon$ gives an isomorphism $V_{Q, x}^{00} \cong V_{Q, x}^{10}$ as unitary spaces and hence

$$
\left(U(V)_{\varepsilon}\right)_{x} \cong \Delta U\left(V_{Q, x}^{00}\right)<\Delta G L\left(V_{Q, x}^{00}\right) .
$$

Case 4. $P=Q Q^{*}$, where $Q$ is an irreducible polynomial, $Q=\operatorname{inv}(Q)$ and $x \neq x^{-1}$.

Note that $G L(V)_{x} \cong \prod_{j} G L\left(V_{Q, x}^{0 j}\right)$ and as before

$$
U(V)_{x} \cong \Delta G L\left(V_{Q, x}^{00}\right)<\left(G L\left(V_{Q, x}^{00}\right)\right)^{2} .
$$

In this case $V_{Q, x}^{0 j}$ and $V_{Q, x}^{1 j}$ are identical as sets and as $F$-vector spaces, but the action of $D_{Q}$ on them differs by a twist by $\mu^{10}$. Hence $\varepsilon$ gives a $\left(D_{Q}, \mu^{10}\right)$ antilinear automorphism of $V_{Q, x}^{0 j}$. Let $W_{j}:=\left(V_{Q, x}^{0 j}\right)^{\varepsilon}$. This is a linear space over $\left(D_{Q}\right)^{\mu^{10}}$. Therefore,

$$
\left(U(V)_{\varepsilon}\right)_{x} \cong \Delta G L\left(W_{0}\right)<\Delta G L\left(V_{Q, x}^{00}\right) .
$$

Case 5. $P=Q Q^{*}$, where $Q$ is an irreducible polynomial, $Q=\operatorname{inv}(Q)$ and $x=x^{-1}$.

As in the previous case,

$$
G L(V)_{x} \cong \prod_{j} G L\left(V_{Q, x}^{0 j}\right) \text { and } U(V)_{x} \cong \Delta G L\left(V_{Q, x}^{00}\right)<\left(G L\left(V_{Q, x}^{00}\right)\right)^{2} .
$$

In this case $D_{Q}=D$ and $\mu^{10}$ is trivial. Hence $V_{Q, x}^{0 j}$ and $V_{Q, x}^{1 j}$ are identical as $D_{Q}$-linear spaces.

Also, $\varepsilon$ gives a $D_{Q}$-linear automorphism of $V_{Q, x}^{0 j}$. So we can interpret $\varepsilon$ as an element in $G L\left(V_{Q, x}^{00}\right)$. Therefore,

$$
\left(U(V)_{\varepsilon}\right)_{x} \cong \Delta\left(G L\left(V_{Q, x}^{00}\right)\right)_{\varepsilon}<\Delta G L\left(V_{Q, x}^{00}\right) .
$$

Case 6. $P$ is irreducible and $x \neq x^{-1}$.

In this case $G L(V)_{x} \cong G L\left(V_{P, x}^{00}\right)$. Also, $V_{P, x}^{00}$ and $V_{P, x}^{01}$ are identical as $F$-vector spaces, but the action of $D_{P}$ on them differs by a twist by $\mu^{01}$. Again, the isomorphism $V_{P, x}^{00} \cong\left(V_{P, x}^{01}\right)^{*}$ gives a $\left(D_{P}, \mu^{01}\right)$ Hermitian structure on $V_{P, x}^{00}$ and

$$
U(V)_{x} \cong U\left(V_{P, x}^{00}\right) \text {. }
$$


Note that $V_{P, x}^{00}$ and $V_{P, x}^{10}$ are identical as $F$-vector spaces, but the action of $D_{P}$ on them differs by a twist by $\mu^{10}$. Hence, $\varepsilon$ gives a $\left(D_{P}, \mu^{10}\right)$ anti-linear automorphism of $V_{P, x}^{00}$. Denote $W:=\left(V_{P, x}^{00}\right)^{\varepsilon}$. It is a linear space over $\left(D_{P}\right)^{\mu^{10}}$. It has a $\left(\left(D_{P}\right)^{\mu^{10}},\left.\mu^{01}\right|_{\left(D_{P}\right)^{\mu^{10}}}\right)$ Hermitian structure. Now

$$
\left(U(V)_{\varepsilon}\right)_{x} \cong U(W)<U\left(V_{P, x}^{00}\right)
$$

Case 7. $P$ is irreducible and $x=x^{-1}$.

Again,

$$
G L(V)_{x} \cong G L\left(V_{P, x}^{00}\right) \text { and } U(V)_{x} \cong U\left(V_{P, x}^{00}\right) .
$$

In this case $D_{P}=D$ and $\mu^{01}=\tau$. Also, $\varepsilon$ commutes with $x$ and hence $\varepsilon \in U(V)_{x} \cong$ $U\left(V_{P, x}^{00}\right)$. Hence

$$
\left(U(V)_{\varepsilon}\right)_{x} \cong U\left(V_{P, x}^{00}\right)_{\varepsilon}<U\left(V_{P, x}^{00}\right)
$$

Case 8. General case.

Let $P=\prod_{i \in I} P_{i}$ be the decomposition of $P$ to irreducible multiples. Since $P$ is proportional to $i n v(P)$, every $P_{i}$ is proportional to $P_{s_{1}(i)}$ for some permutation $s_{1}$ of $I$ of order $\leq 2$. Since $P$ is proportional to $P^{*}$, every $P_{i}$ is proportional to some $P_{s_{2}(i)}$. This gives rise to an action of $\mathbb{Z} / 2 \mathbb{Z} \times \mathbb{Z} / 2 \mathbb{Z}$ on $I$.

Let $I=\bigsqcup I_{\alpha}$ be the decomposition of $I$ to orbits of this action. Denote $V_{\alpha}:=$ $\operatorname{Ker}\left(\prod_{i \in \alpha} P_{i}(x)\right)$. Clearly $V=\bigoplus V_{\alpha}$ and $V_{\alpha}$ are orthogonal to each other and $\varepsilon$-invariant. Hence the pair $\left(U(V)_{x},\left(U(V)_{\varepsilon}\right)_{x}\right)$ is a product of pairs from the first 7 cases.

6.7. Genealogical trees of the symmetric pairs considered in this paper. The following diagrams sum up the results of this section.

An arrow " $\left(G_{1}, H_{1}\right) \rightarrow\left(G_{2}, H_{2}\right)$ " means that pairs of type $\left(G_{1}, H_{1}\right)$ may have descendants with factors of the type $\left(G_{2}, H_{2}\right)$. We will not draw the obvious arrows " $(G, H) \rightarrow(G, H)$ " and when we draw " $\left(G_{1}, H_{1}\right) \rightarrow\left(G_{2}, H_{2}\right) \rightarrow\left(G_{3}, H_{3}\right)$ " we mean also " $\left(G_{1}, H_{1}\right) \rightarrow\left(G_{3}, H_{3}\right)$ ".

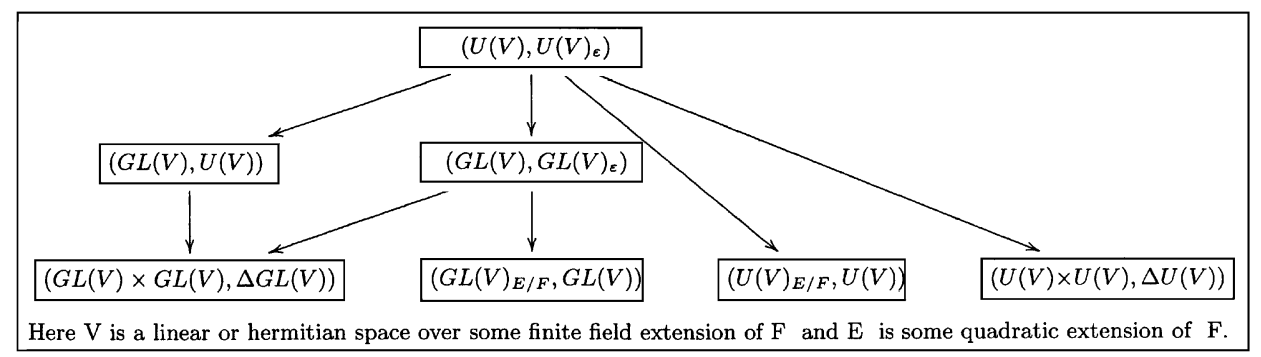




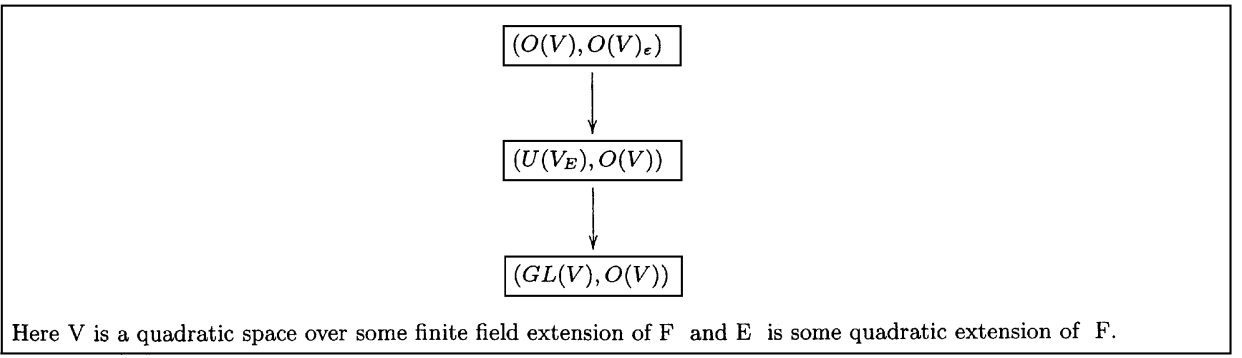

\section{ACKNOWLEDGEMENTS}

We are grateful to Herve Jacquet for a suggestion to consider the pair $\left(U_{2 n}\right.$, $\left.U_{n} \times U_{n}\right)$ which inspired this paper. We also thank Joseph Bernstein, Erez Lapid, Eitan Sayag and Lei Zhang for fruitful discussions.

\section{REFERENCES}

[AG1] A. Aizenbud, D. Gourevitch, Schwartz functions on Nash Manifolds, Int. Math. Res. Not. IMRN 2008, no. 5, Art. ID rnm 155, 37 pp. MR2418286

[AG2] A. Aizenbud, D. Gourevitch (with an appendix by A. Aizenbud, D. Gourevitch and E. Sayag): Generalized Harish-Chandra descent, Gelfand pairs and an Archimedean analog of Jacquet-Rallis' Theorem, postprint: arXiv:0812.5063v4[math.RT]. Originally published in: Duke Mathematical Journal, Volume 149, Number 3, pp. 509-567 (2009).

[AGS] A. Aizenbud, D. Gourevitch, E. Sayag: $\left(\mathrm{GL}_{n+1}(F), \mathrm{GL}_{n}(F)\right)$ is a Gelfand pair for any local field F, postprint: arXiv:0709.1273v4[math.RT]. Originally published in: Compositio Mathematica, 144, pp. 1504-1524 (2008), doi:10.1112/S0010437X08003746.

[BvD] E. E H. Bosman and G. Van Dijk, A New Class of Gelfand Pairs, Geom. Dedicata 50 (1994), no. 3, 261-282. MR.1286380 (95j:22025)

[BZ] J. Bernstein, A.V. Zelevinsky, Representations of the group $\operatorname{GL}(n, F)$, where $F$ is a local non-Archimedean field, Uspekhi Mat. Nauk 31 (1976), No.3, 5-70. MR0425030(54:12988)

[GK] I.M. Gelfand, D. Kazhdan, Representations of the group $\operatorname{GL}(n, K)$ where $K$ is a local field, Lie groups and their representations (Proc. Summer School, Bolyai Janos Math. Soc., Budapest, 1971), pp. 95-118. Halsted, New York (1975). MR0404534 (53:8334)

[Gro] B. Gross, Some applications of Gelfand pairs to number theory, Bull. Amer. Math. Soc. (N.S.) 24 (1991), no. 2, 277-301. MR.1074028 (91i:11055)

[MVW] Colette Møglin, Marie-France Vigneras, Jean-Loup Waldspurger, Correspondances de Howe sur un corps p-adique. (French) [Howe correspondences over a p-adic field] Lecture Notes in Mathematics, 1291, Springer-Verlag, Berlin, 1987. viii+163 pp. MR 1041060 (91f:11040)

[SpSt] T. A. Springer, R. Steinberg, Conjugacy classes. 1970 Seminar on Algebraic Groups and Related Finite Groups (The Institute for Advanced Study, Princeton, N.J., 1968/69) pp. 167-266. Lecture Notes in Mathematics, Vol. 131, Springer, Berlin. MR0268192 (42:3091)

[vD] G. van Dijk, On a class of generalized Gelfand pairs, Math. Z. 193 (1986), 581-593. MR0867349 (87m:22041)

Faculty of Mathematics and Computer Science, The Weizmann Institute of Science, POB 26, REHOVOT 76100, ISRAEL

E-mail address: aizenr@yahoo.com

Faculty of Mathematics and Computer Science, The Weizmann Institute of Science, POB 26, Rehovot 76100, IsRAel

Current address: School of Mathematics, Institute for Advanced Study, Einstein Drive, Princeton, New Jersey 08540

E-mail address: guredim@yahoo.com 